Instituto Internacional de Investigación y Desarrollo Tecnológico Educativo INDTEC, C.A.

DOI: https://doi.org/10.29394/Scientific.issn.2542-2987.2020.5.16.18.334-351

OAI-PMH: http://www.indteca.com/ojs/index.php/Revista Scientific/oai

Ensayo Original / Original Essay

\title{
Las tecnologías en la organización de un aula inclusiva para niños con capacidades especiales
}

\author{
Autores: Miriam Alexandra Heras Rojas \\ Universidad Nacional de Educación, UNAE \\ alexandraheras2015@gmail.com \\ Azogues, Ecuador \\ https://orcid.org/0000-0002-3178-5731 \\ Rodrigo Mauricio Orden Verdugo \\ Universidad Nacional de Educación, UNAE \\ mauricio.ordenv@gmail.com \\ Azogues, Ecuador \\ https://orcid.org/0000-0002-5770-0409 \\ Valeria Jacqueline Serrano Castro \\ Universidad Nacional de Educación, UNAE \\ jserranoc89@gmail.com \\ Azogues, Ecuador \\ https://orcid.org/0000-0001-9420-1812
}

\section{Resumen}

El presente ensayo, aborda como la atención a la diversidad dentro de un aula inclusiva, puede generar aprendizajes significativos, utilizando las TIC, siendo la inclusión un compromiso básico en la construcción de una educación de calidad como señala Parrilla (2004), destacando que el aprendizaje es un derecho y una posibilidad de participación en el proceso de aprendizaje. Este enfoque pretende llevar a cabo una verdadera integración de todos los estudiantes atendiendo a la diversidad como propone Fernández y Bermejo (2012), mediante actitudes positivas de los docentes y una adecuada utilización de las TIC, que brinden una mejor atención a niños con Necesidades Educativas Especiales asociadas o no a la discapacidad. El uso de las TIC genera muchas ventajas en el campo educativo, pero también, algunas barreras u obstáculos que dificultan o impiden una igualdad de oportunidades, como lo destacan Corrales, Soto y Villafañe (2016). Estas barreras serán superadas con una gestión educacional que fusione a todos los actores educativos, donde el papel del docente sea innovador utilizando todas las herramientas que estén a su alcance entre ellas las tecnológicas, para que los estudiantes desarrollen sus habilidades y destrezas desde sus particularidades y que así aporten aún más, a toda la sociedad.

Palabras clave: gestión educacional; papel del docente; necesidades educativas.

Cómo citar este ensayo:

Heras, M., Orden, R., \& Serrano, V. (2020). Las tecnologías en la organización de un aula inclusiva para niños con capacidades especiales. Revista Scientific, 5(16), 334-351, e-ISSN: 2542-2987. Recuperado de: https://doi.org/10.29394/Scientific.issn.2542-2987.2020.5.16.18.334-351

Fecha de Recepción: 12-12-2019
Fecha de Aceptación: 16-03-2020
Fecha de Publicación: 05-05-2020 
Ensayo Original / Original Essay

\title{
Technologies in the organization of an inclusive classroom for children with special abilities
}

\begin{abstract}
This essay addresses how attention to diversity within an inclusive classroom can generate significant learning, using ICT, with inclusion being a basic commitment in the construction of quality education, as Parrilla (2004), points out, highlighting that the Learning is a right and a possibility of participation in the learning process. This approach aims to carry out a true integration of all students attending to diversity as proposed by Fernández and Bermejo (2012), through positive attitudes of teachers and an adequate use of ICT, which provide better care for children with Educational Needs Special associated or not with disability. The use of ICT generates many advantages in the educational field, but also, some barriers or obstacles that hinder or impede equal opportunities, as highlighted by Corrales, Soto and Villafañe (2016). These barriers will be overcome with an educational management that merges all educational actors, where the role of the teacher is innovative using all the tools available to them, including technological ones, so that students develop their abilities and skills from their particularities and so that they contribute even more to the whole of society.
\end{abstract}

Keywords: educational management; teacher role; educational needs.

How to cite this essay:

Heras, M., Orden, R., \& Serrano, V. (2020). Technologies in the organization of an inclusive classroom for children with special abilities. Revista Scientific, 5(16), 334-351, e-ISSN: 2542-2987. Recovered from: https://doi.org/10.29394/Scientific.issn.2542-2987.2020.5.16.18.334-351

Date Received:

12-12-2019
Date Acceptance:

16-03-2020
Date Publication: 05-05-2020 


\section{Introducción}

Hablar de capacidades especiales es adentrarse a un tema muy amplio y profundo de análisis y conversación, en este sentido necesitaríamos un sinnúmero de especialistas para abordar punto por punto y paso a paso cada capacidad especial que se encuentre dentro del sistema educativo. Un sistema educativo que en sí presenta problemas en el proceso de enseñanza aprendizaje para poder llegar a todos los estudiantes, más aún cuando se atiende a una diversidad que debe ser incluida en todo sentido y a todo nivel.

Frente a esto, Holzschuher (2012): habla de "Cómo organizar aulas inclusivas ofrece ideas y estrategias para abordar y planificar un aula escolar" (pág. 7); se mencionan operaciones que se deben tener en cuenta para un aula inclusiva, ahora nos planteamos la pregunta ¿un aula es más inclusiva con el apoyo de la tecnología? Esta interrogante podría ser fácilmente respondida, pero al momento que contextualizamos las realidades institucionales de varios establecimientos, se puede notar que no es tan sencillo la implementación de la tecnología en el proceso educativo por varios factores.

En este ensayo trataremos de abordar el cómo podemos utilizar las tecnologías y que tanto conocemos de estas, para que mediante aulas inclusivas podamos mejorar el proceso de enseñanza aprendizaje a la diversidad de las comunidades educativas, así como a los actores del entorno educativo con capacidades especiales.

\section{Desarrollo}

\subsection{Educación Inclusiva y tecnologías}

Desarrollar una educación inclusiva concebida como un proceso, permite abordar y responder a la diversidad de necesidades que involucren a toda la comunidad educativa, esto implica una serie de ajustes y modificaciones en el aula y más aún en el sistema educativo que hasta la 
actualidad, se muestra limitada por falta de conocimientos, estrategias metodológicas, actitudes, intereses y predisposiciones por parte de los docentes a la hora de desempeñarse en su labor educativa, que de acuerdo con, Parrilla (2004), es:

Un compromiso básico e irrenunciable en el planteamiento inclusivo es la construcción de una educación de calidad, para todos, lo que supone pensar en el aula ordinaria como espacio de aprendizaje instructivo para todos los alumnos, en el que el aprendizaje es un derecho y una posibilidad que ha de concretarse en la participación en el proceso de aprendizaje (pág. 5).

En este sentido, si la educación fuera en realidad humanista y no un proceso repetitivo de conocimientos, que muchas de las veces ya resultan hasta caducos, debido a lo mecánicos que se han convertido muchos procesos de enseñanza aprendizaje, manejados por algunos docentes y hasta instituciones, la inclusión estaría presente en el día a día de nuestro sistema educativo, de manera práctica, enfocada a la atención a la diversidad, aprovechando las diferencias de manera productiva y positiva.

Con el fin de conseguir la igualdad de oportunidades entre estudiantes, Araque y Barrio (2010), indican que es importante prestar especial atención a:

a) Características generales: Estilos cognitivos; Ritmos de aprendizaje; Intereses y motivaciones. b) Diferencia de capacidades: Altas capacidades; Discapacidades (motoras, psíquicas, sensoriales, de personalidad). c) Diferencias sociales: Desarrollo en entornos sociales desfavorecidos; Pertenencia a minorías étnicas o culturales; Desconocimiento de la lengua mayoritaria o vehicular del sistema; Itinerancia; Hospitalización y convalecencia (pág. 12).

Evidentemente, este enfoque propone realizar ajustes 0 transformaciones en la organización de las aulas de manera adecuada, motivadora y divertida para llegar a cada alumno, partiendo de las potencialidades, habilidades y necesidades de este, con el objetivo de 
aprovechar los recursos tecnológicos que tenemos a nuestro alcance.

La idea de transformar las aulas inclusivas con un abnegado compromiso docente, introduciendo el buen uso de las tecnologías en la educación, tomando en cuenta la diversidad educativa y brindando atención a sus necesidades, posibilita un avance significativo, incorporando el conocimiento productivo en el proceso de mejora e innovación con recursos audiovisuales que requieran más alternativas innovadoras.

Por lo que, Hinojo y Fernández (2002), citados por Fernández y Bermejo (2012), destacan que:

Es en esa transformación de las acciones docentes donde la actitud del profesorado frente a la utilización de las TIC constituye un aspecto clave unido a otros como las competencias y los medios de que disponen para poder llevar a cabo esta integración. A pesar de ello, todavía son muchos los profesionales de la educación que no han desarrollado una actitud favorable hacia las TIC en el marco de la atención a la diversidad. Ésta puede ser una de las razones por las que no las utilicen en el aula, lo que conlleva una falta de aprovechamiento de los beneficios que estas tecnologías pueden aportar, tanto a su trabajo como al aprendizaje de sus alumnos (pág. 48).

Si bien es cierto, que la implementación de las Tecnologías en la Educación Inclusiva, se ha convertido en un pilar fundamental en nuestros días, facilitando el acceso a la información y comunicación, potenciando en los estudiantes los conocimientos de manera complementaria, pues evidentemente el adecuado uso de las Tecnologías transforma los entornos educativos de los estudiantes con alguna necesidad para hacerlos más inclusivos, ya que tiene la capacidad de adaptarse y contribuir con aprendizajes significativos, favoreciendo la atención personalizada de todos los estudiantes y alcanzando óptimos resultados en su proceso de manera productiva, que todos alcancen al máximo el desarrollo del conocimiento sin escatimar a ningún educando de manera equitativa en cualquier contexto. 
Instituto Internacional de Investigación y Desarrollo Tecnológico Educativo INDTEC, C.A.

DOI: https://doi.org/10.29394/Scientific.issn.2542-2987.2020.5.16.18.334-351

OAI-PMH: http://www.indteca.com/ojs/index.php/Revista_Scientific/oai

Ensayo Original / Original Essay

De esta manera, señala Morín (1998), citado por Domínguez, et al. (2018):

La tecnología ha contribuido de muchas formas a las personas que tienen necesidades especiales para lograr su integración a la sociedad. Las herramientas tecnológicas han sido de gran apoyo para igualar sus oportunidades en la vida personal, laboral, social e incluso en el ámbito académico así fundamentando una sociedad justa (pág. 306).

El desafío docente de pertinencia y prospectiva se considera radicalmente necesario, hacer hincapié en reorganizar las aulas educativas, en consideración a las herramientas tecnológicas; pues, tienen la capacidad de adaptarse a estudiantes con Necesidades Educativas Especiales, asociados o no la discapacidad, desarrollando en los estudiantes la construcción de su propio conocimiento de manera significativa e innovadora apegándose a quienes tengan más dificultades, tomando en cuenta sus necesidades así como también sus habilidades, facilitando la información y la comunicación a fin de brindar mayor apertura y adentrarse en el proceso educativo, facilitando la adquisición del aprendizaje como una oportunidad de poder interactuar, pues el uso de la tecnología puede convertirse en una oportunidad para todos, de tal forma que se lleve con éxito el aprendizaje, a fin de que nos encontremos estrechamente vinculados con la tecnología y su avance en la educación inclusiva.

De acuerdo con Román, Cardemil y Carrasco (2011), citados por Rodríguez y Arroyo (2014), destacan que:

La incorporación de las TIC en el trabajo de los alumnos/as con necesidades educativas especiales, permiten indudablemente un mayor acceso a la información, la comunicación y la cultura por parte de los mismos, siendo "el medio que posibilita la inclusión e integración a las sociedades, al tiempo que se constituyen en potentes herramientas didácticas para fortalecer capacidades y habilidades propias de los aprendices del nuevo milenio..." (pág. 111). 
Por ello, es de fundamental importancia el compromiso docente a fin de organizar y reorganizar las aulas; de manera que, la educación inclusiva ya no se vea sólo como una meta, sino más bien poderla ver como una realidad cumplida atendiendo a las necesidades e intereses de los estudiantes.

En este sentido, implementar esta visión de pertinencia humanista, donde basados en una ética profesional y social cuidemos del otro con respeto, valorando todas sus capacidades y cualidades, estaríamos avanzando hacia una educación inclusiva vinculada a la atención a la diversidad que difundan paradigmas de comportamiento social y actitudinal, apoyándonos en los recursos tecnológicos, promoviendo la integración y estimulando el desarrollo de habilidades intelectuales.

\subsection{Las barreras de la tecnología en la educación especial}

En pleno Siglo XXI la inserción de tecnologías en la educación es un tema todavía preocupante, pues a pesar de conocer su objetivo, el mismo que es mejorar el proceso educativo, todavía existen barreras que impiden la aplicación de estas.

Como plantea De Asís Roig (2005), citado por Corrales, Soto y Villafañe (2016a), la barrera se refiere a: "todo obstáculo que dificulte o impida, en condiciones de igualdad de oportunidades y de plena participación, el acceso de las personas a alguno/s de los ámbitos [...]" (pág. 4); en efecto, podemos decir que las barreras son un impedimento para alcanzar los objetivos planteados y en muchas ocasiones el currículo en sí, que como menciona, Echeita (2013): "dificulta las dinámicas de pertenencia, participación y reconocimiento en la vida escolar de determinados alumnos, así como un serio impedimento para promover la igualdad de oportunidades de aprendizaje" (pág. 107-108); por lo tanto, las barreras educativas parten del contexto en donde se desenvuelve el estudiante, convirtiéndose en fuente principal de desventaja para el mismo. 
En esa misma línea, Montigny (2010), citado por Corrales, Soto y Villafañe (2016b): hace referencia a la accesibilidad como un principio para "promover la integración de las personas con discapacidad en todos los aspectos de la sociedad" (pág. 5); en este caso es necesario resaltar que accesibilidad no solo trata del espacio físico si no en general, es decir, en el apoyo social tanto de familia como docentes, siendo así que se debe buscar posibles alternativas para su eliminación y/o modificar las barreras para lograr la integración total al mismo.

Son varios los aspectos, tanto internos como externos, que se podrían considerar barrera en la aplicación de la tecnología en la educación especial, las mismas que deben ser superadas en su totalidad, de tal suerte que no se quede en la mera novedad de aplicar nuevas herramientas sino por el contrario que su uso sea efectivo.

\subsection{Capacidades especiales en el país}

En virtud de los puntos tratados, para contextualizarlos en cómo aportan las tecnologías en el aprendizaje de niños con capacidades especiales, se presentan las cifras con corte a febrero del año 2020, por parte del Consejo Nacional de Igualdad de Discapacidades (CONADIS, 2020): centrándose en la población de 4 a 18 años que son las edades consideradas desde el nivel de inicial hasta tercero de bachillerato aproximadamente, existen 64933 niños, niñas y/o adolescentes con alguna discapacidad registrados a nivel del país, según el tipo de discapacidad tenemos los siguientes porcentajes: $28,68 \%$ física, $51,68 \%$ intelectual, $8,52 \%$ auditiva, $4,80 \%$ visual, 6,32\% psicológico, como se expresa en el gráfico 1. 
Instituto Internacional de Investigación y Desarrollo Tecnológico Educativo INDTEC, C.A.

DOI: https://doi.org/10.29394/Scientific.issn.2542-2987.2020.5.16.18.334-351

OAI-PMH: http://www.indteca.com/ojs/index.php/Revista_Scientific/oai

Ensayo Original / Original Essay

Gráfico 1. Porcentajes por tipo de discapacidad a nivel país.

Tipo de Discapacidad

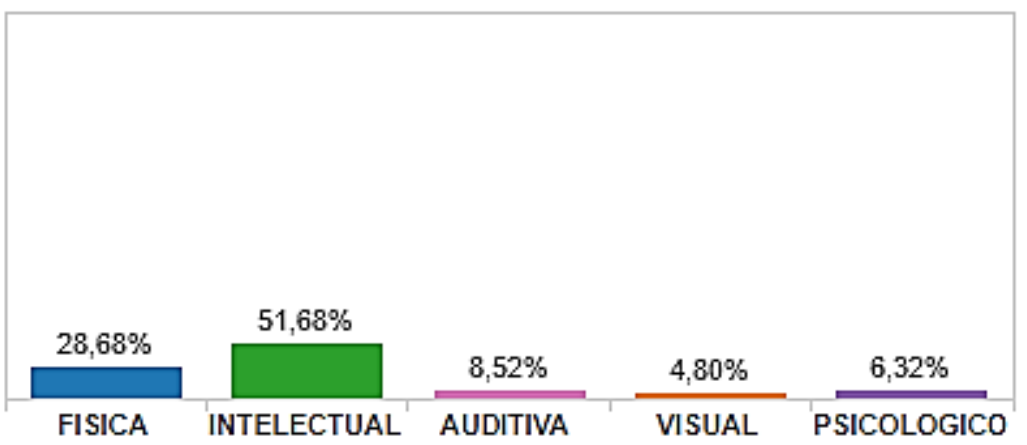

Fuente: Consejo Nacional para la Igualdad de Discapacidades (2020).

Según los grupos etarios seleccionados tenemos los siguientes porcentajes: 9,24\% de 4 a 6 años, $37,61 \%$ de 7 a 12 años y $53,15 \%$ de 13 a 18 años, expuesto en el gráfico 2. Con estos datos podemos observar que el mayor número de estudiantes con capacidades especiales estarían ubicados en los niveles de octavo de básica a tercero de bachillerato, con estos números sería de vital importancia a nivel país considerar la aplicación de estrategias para aulas y sistemas inclusivos en estos niveles de educación básica superior y bachillerato, el cual resulta un poco coherente, ya que en casi todas las instituciones es en el octavo de básica en donde los estudiantes pasan de tener un docente de aula a varios docentes por materias y/o áreas.

Gráfico 2. Grupos Etarios a nivel país.

\section{Grupos Etarios}

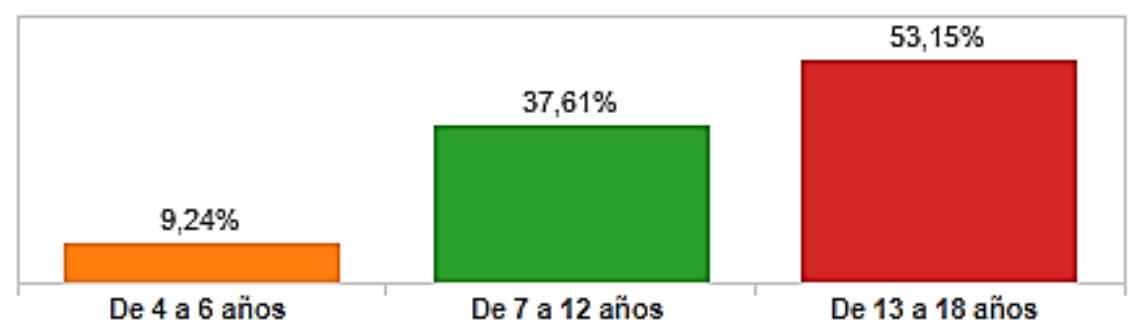

Fuente: Consejo Nacional para la Igualdad de Discapacidades (2020). 
Dentro de la provincia del Cañar, en específico el cantón Azogues, el CONADIS presenta 516 niños, niñas y/o adolescentes entre los 4 y los 18 años, con los siguientes porcentajes de discapacidad: 13,57\% física, $69,19 \%$ intelectual, $6,20 \%$ auditiva, $4,07 \%$ visual y $6,98 \%$ psicológica, como se expresa en el gráfico 3 .

Gráfico 3. Porcentajes por tipo de discapacidad cantón Azogues.

Tipo de Discapacidad

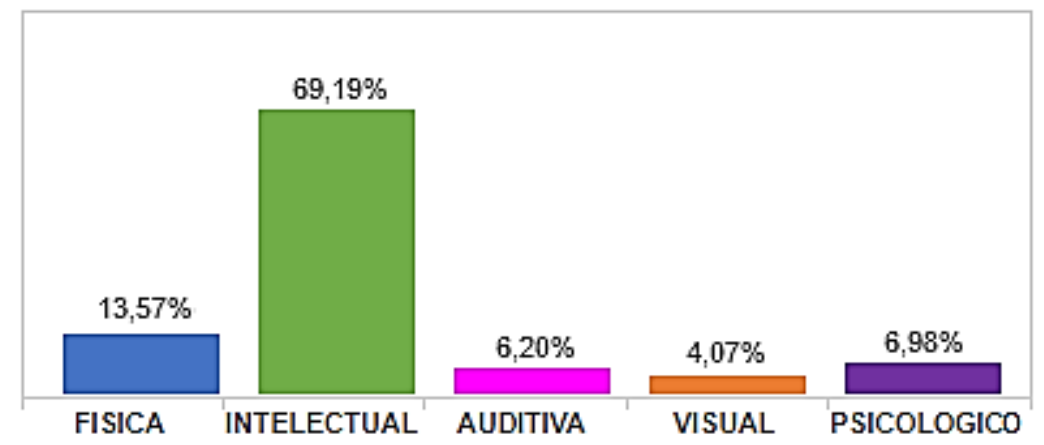

Fuente: Consejo Nacional para la Igualdad de Discapacidades (2020).

En el gráfico 4, podemos observar los porcentajes según los grupos etarios que presenta el cantón Azogues en febrero del año 2020, con 6,98\% en el grupo de 4 a 6 años, 39,53\% en el grupo de 7 a 12 años y 53,49\% en el grupo de 13 a 18 años. Estos datos indican que, en el cantón al igual que en el país, el mayor porcentaje de discapacidad se ubica en las edades que cursan desde octavo de básica hasta tercero de bachillerato.

Gráfico 4. Grupos Etarios cantón Azogues.

\section{Grupos Etarios}

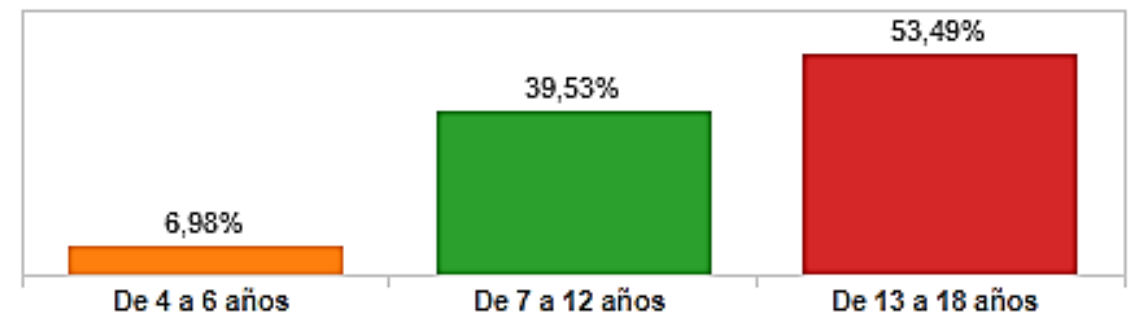

Fuente: Consejo Nacional para la Igualdad de Discapacidades (2020). 
Los datos analizados en el mes de febrero del año 2020, indican que existen varios tipos de capacidades especiales en nuestro país, si bien es cierto el currículo nacional habla de inclusión de los niños con Necesidades Educativas Especiales (NEE) en el sistema de educación ordinario, todavía se deben dar muchos pasos para que esto sea una realidad. La inclusión aún no se alcanza y estamos en el proceso de adaptarnos a las tecnologías que avanzan a pasos agigantados para que sean una herramienta para esta atención a la diversidad anhelada.

\subsection{Contextualizando la tecnología en la atención a las capacidades especiales}

Con el objeto de palpar más de cerca cómo se encuentra el proceso del uso de tecnologías en el proceso de enseñanza aprendizaje a niños con capacidades especiales, se realizó una encuesta a 18 docentes de la Unidad de Educación Especial "Manuela Espejo" ubicada en la ciudad de Azogues, en la cual, se ha podido obtener información sobre posibles barreras existentes en la institución para la aplicación de las diferentes tecnologías educativas en las misma.

A continuación, se explicará los resultados más relevantes según la opinión obtenida de cada pregunta, la misma se divide en tres barreras obtenidas de la tabulación.

\subsubsection{Falta de formación}

La primera barrera dentro del sistema nacional de educación especial, es la escasa dotación de software y hardware a los centros educativos, y los pocos que se cuentan (computadoras y proyectores multimedia) no corresponden a una infraestructura idónea y acorde a las necesidades de los estudiantes que se forman en el plantel. El sistema educativo debe responder eficientemente a las capacidades, ritmo de aprendizaje y demás 
características propias de la población estudiantil.

Dentro de la institución no existe un conocimiento sobre el concepto de las TIC, ya que su concepto está enfocado solo como el uso del internet, cabe recalcar que TIC son las Tecnologías de la Información y la Comunicación, ya que están involucradas todas las herramientas que nos ayuden a una mejor comprensión de los conocimientos tales como pueden ser radio, televisión, tabletas, entre otras.

Otro punto relevante es que las tecnologías nos hacen dependientes y pocos reflexivos, partiendo de la falta de conocimiento en el uso de las tecnologías, ya que si se utilizase de la manera adecuada se puede aprovechar la potencialidad de los alumnos y se ayuda en el comprensión, reflexión y análisis partiendo de la realidad y capacidad de los alumnos.

Complementando lo anterior, no existe un interés por parte de las docentes en conocer nuevas aplicaciones que ayuden a una mejor comprensión y aplicación del proceso enseñanza aprendizaje.

\subsubsection{Poco hábito en el uso de las TIC}

La segunda barrera es la falta de preparación del profesorado en el ámbito del uso las tecnologías en ambientes escolares. No basta con saber manjares utilitarios, navegar en internet, etc., el éxito radica en saber aplicar las tecnologías en el aula y más todavía si se trata de personas de inclusión, quienes generalmente están acostumbrados a una rutina académica muy pasiva.

Partiendo del análisis de la encuesta se ha podido evidenciar que según las docentes no hacen uso de la tecnologías frecuentemente, pero cabe recalcar que al momento de utilizar las herramientas como videos, música entre otros, se está haciendo el uso del mismo, esta falencia se da por falta de información, falta de capacitación sobre el uso y las diferentes tecnologías existentes en el medio que se pueden utilizar para un mejor proceso 
enseñanza aprendizaje, convirtiéndose así en una barrera general dentro de la institución.

\subsubsection{Desigualdades de acceso}

La tercera barrera es la brecha digital, que, en nuestro país, hasta la actualidad es un problema latente, más todavía en la población que pertenece a los quintiles de pobreza, que en el caso particular de la Unidad Educativa Especial "Manuela Espejo" corresponde a un importante porcentaje, lo que imposibilita que los estudiantes refuercen los conocimientos en sus casas y menos aún van a recibir un apoyo o refuerzo de parte de sus familiares y la gran diversidad de disparidades que el plantel atiende, lo que demandaría la existencia de varias tecnologías acopladas a la condición a cada estudiante.

De acuerdo con los resultados de las encuestas se ha evidenciado que dentro de la institución no cuenta con las herramientas necesarias para todo el cuerpo docente, y si presentan algún tipo de herramienta es por parte personal que lo han adquirido, de igual manera las capacitaciones han sido realizadas por parte personal de cada docente, más no por parte de la institución o de los superiores.

\section{Conclusiones}

En consecuencia, la organización de un aula inclusiva que responda a los intereses de los estudiantes con Necesidades Educativas Especiales está en nuestras manos, por lo tanto es de fundamental importancia aplicar de manera adecuada el buen uso de las Tecnologías de tal forma que se logre la interacción educativa y la construcción de su propio conocimiento de manera productiva y transformadora, brindando atención y respuestas a las necesidades de los estudiantes a fin de lograr aprendizajes significativos, desarrollando en los estudiantes su capacidad de generar un pensamiento crítico, su autonomía y su adaptación dentro del entorno educativo, en donde 
los estudiantes se sientan felices de poder compartir sus experiencias, por ello es tarea de los docentes ejecutar este gran reto a fin de promover una educación de calidad y calidez.

Una educación que sea capaz de vencer las barreras que se presenten y generar estrategias con apoyo de la tecnología para la enseñanza de los educandos, vinculando a toda la comunidad educativa para educar con y en la diversidad, contextualizando los programas a las realidades de cada institución y dando el valor al docente, para que se empodere de los procesos educativos y se convierta en ese docente investigativo, innovador y creador de espacios atractivos de aprendizaje para cada uno de sus estudiantes con y en la realidad actual que nos engloba este mundo tecnológico en el que nos desenvolvemos a diario.

\section{Referencias}

Araque, N., \& Barrio, J. (2010). Atención a la diversidad y desarrollo de procesos educativos inclusivos. Prisma Social, (4),1-37, e-ISSN: 1989-3469. Recuperado de:

https://dialnet.unirioja.es/servlet/articulo?codigo $=3632700$

Corrales, A., Soto, V., \& Villafañe, G. (2016a,b). Barreras de aprendizaje para estudiantes con discapacidad en una universidad chilena. Demandas estudiantiles y desafíos institucionales. Actualidades Investigativas en Educación, 16(3), 1-29, e-ISSN: 1409-4703. Recuperado de: https://doi.org/10.15517/aie.v16i3.25957

CONADIS (2020). Estadísticas de Discapacidad. Quito, Ecuador: Consejo Nacional para la Igualdad de Discapacidades. Recuperado de: https://www.consejodiscapacidades.gob.ec/estadisticas-dediscapacidad/

Domínguez, J., Guarda, T., Torres, W., Bustos, S., Villao, F., Sánchez, J., ... Ponce, V. (2019). Herramientas Tecnológicas Contribuyendo a la 
Inclusión de Personas con Necesidades Especiales. risti: Revista Ibérica de Sistemas e Tecnologias de Informação, (E18), 302-309, eISSN: 1646-9895. Recuperado de:

http://www.risti.xyz/issues/ristie18.pdf

Echeita, G. (2013). Inclusión y exclusión educativa. De nuevo "Voz y Quebranto". REICE. Revista Iberoamericana sobre Calidad, Eficacia y Cambio en Educación, 11(2), 99-118, e-ISSN: 1696-4713. Recuperado de: https://www.redalyc.org/pdf/551/55127024005.pdf

Fernández, J., \& Bermejo, B. (2012). Actitudes docentes hacia las TIC en centros de buenas prácticas educativas con orientación inclusiva. Enseñanza \& Teaching, 30(1), 45-61, e-ISSN: 0212-5374. Recuperado de: https://revistas.usal.es/index.php/0212-5374/article/view/9296

Holzschuher, C. (2012). Cómo organizar aulas inclusivas: Propuestas y estrategias para acoger las diferencias. Madrid, España: Narcea Ediciones.

Parrilla, M. (2004). La construcción del aula como comunidad de todos. Organización y gestión educativa, 12(2), 19-29, ISSN: 1134-0312. España: Fórum Europeo de Administradores de la Educación.

Rodríguez, M., \& Arroyo, M. (2014). Las TIC al servicio de la inclusión educativa. Digital Education Review, (25), 108-126, e-ISSN: 20139144. Recuperado de:

https://dialnet.unirioja.es/servlet/articulo?codigo $=4778259$ 


\section{Ensayo Original / Original Essay}

\section{Miriam Alexandra Heras Rojas}

email: alexandraheras2015@gmail.com

Nacida en Cojitambo, Azogues, Ecuador, el 4 de mayo del

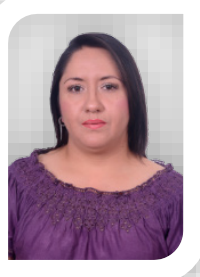
año 1985. Licenciada en Ciencias de la Educación Mención Educación Parvularia; Especialista en Docencia Universitaria; Docente de la Unidad Educativa Particular Universitaria de Azogues, campus de la Universidad Católica de Cuenca (UCACUE), Sede Azogues, en el periodo 2008-2018; actualmente me desempeño como Profesora de Educación Básica Media en la Unidad Educativa Juan Bautista Vásquez, en Azogues, Ecuador, desde el año 2018; Maestrante del Programa de Educación Inclusiva. 
Ensayo Original / Original Essay

\section{Rodrigo Mauricio Orden Verdugo email: mauricio.ordenv@gmail.com}

Nacido en Cuenca, Ecuador, el 7 de enero del año 1984.

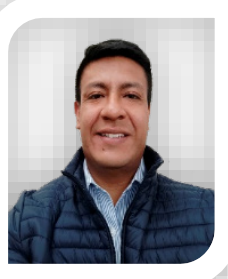
Ingeniero Mecánico con mención en Diseño de Máquinas de la Universidad Politécnica Salesiana (UPS); Docente de la Unidad Educativa Sinincay en la especialidad de Mecanizado y Construcciones Metálicas Aplicadas, desde el periodo 2009 hasta la actualidad. 


\section{Valeria Jacqueline Serrano Castro \\ e-mail: jserranoc89@gmail.com}

Nacida en Azogues, Ecuador, el 10 de mayo del año 1989.

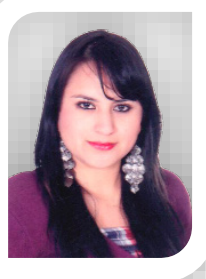
Los estudios primarios los realicé en la escuela "Dolores Sucre", el bachillerato en el colegio "Luis Cordero" y los estudios superiores en la Universidad del Azuay (UDA), en donde obtuve el título de Licenciada en Ciencias de la Educación mención Especial y Básica; me he desempeñado como tutora particular de niños con necesidades educativas especiales en proceso de inclusión en el sistema regular, desde el periodo 2013-2018; laboré como docente en la Unidad Educativa Particular Universitaria de Azogues, campus de la Universidad Católica de Cuenca (UCACUE), Sede Azogues, en donde integré el consejo ejecutivo en el periodo 2016-2018; en septiembre del año 2018 hasta la actualidad, ejerzo labores de docente en la Unidad Educativa Especial "Manuela Espejo".

El contenido de este manuscrito se difunde bajo una Licencia de Creative Commons ReconocimientoNoComercial-Compartirlgual 4.0 Internacional 